\title{
FERAL ROBOTIC BIRDS
}

Lou Terry ${ }^{1}$

Goldsmiths, London, United Kingdom

This paper will ask what potential the frameworks of actor-network theory and multispecies storytelling have for informing our understanding of environmental change. Specifically, it will explore how combining these two theories might reveal the web of entanglements between us and our environments, alongside our joint environmental citizenship with our non-human counterparts. It will then look at how we might sense and articulate environments in such a way as to render these entanglements and joint environmental citizenship visible (and audible). Through research led practice, the paper explores the implications that engagement with non-human organisms, specifically through sound - with an ear to artistic modes of perception as well as scientific - have for forming critical insights into environmental and ecological change.

Keywords: actor network theory, multispecies, environmental media, environmental sensing, soundscape ecology, birdsong

The ancients observed birds erupting from spaces in the sky and invented letters from the shapes they described. the birds, in return, insisted on the possibility of song (Sean Bonney - On the relationship of hermits to the means of information) 


\section{INTRODUCTION}

Since the 19th Century, observational sciences have represented the world through "precision measurement, numerical data processing, and the representation of scientific information on a global or cosmic scale", Gabrys (2013: 133). The recording of such observation and measurement has formed useful data repositories, from which environmental monitoring has been able to draw correlations between data over time, and explain unified geological concepts. Through these methods, objects that the data represents can be seen to be causative, forming a topological understanding of environmental dynamics, containing a root source, or unified explanation, of certain phenomena. More recently, climate change has steered the figuring of environmental change towards a root cause, namely greenhouses gases, particularly $\mathrm{CO}_{2}$. Observational science has gone a long way in revealing how the absorption by greenhouse gases of solar radiation reflecting from earth is connected to increased tropospheric air and sea temperatures, melting sea ice and glaciers, increased sea level rise, habitat change and destruction, and loss of biodiversity. Through observational science, molecules, previously invisible to us, have been revealed as global actors of adverse environmental changes for much of life on earth (and also, paradoxically, why life can exist here at all).

Going forward however, it is important to interrogate how the ways in which we figure environmental changes govern our approaches to environmental challenges. For example, the observing of greenhouse gases as the source, or cause of global warming, has presented the atmosphere as an exterior entity; one which we need to act on, or fix. This has led to the development of technological solutions, ranging from electric cars, to renewable energy projects and even weather control systems. However, these technologies can fail to address, or draw our attention to, the myriad agents and multiple sources of environmental change (such as our own behaviour), which are both multi-dimensional and mutually entangled with the web of historic apparatus that forms the nature and culture we swim in. When we think of technologies we often think just of the intent, or dream of the technology, Tsing (2016). An electric car may be something that drives on electricity, but how do we account for the collateral - the systems of underpaid labour and environmental degradation of mining lithium for car batteries, the previous cars thrown away and now rusting in dumps, or markets cultivating a cultural preference for private travel over public modes of transport? These kinds of challenges, existing on the periphery of many technological fixes to environmental change, are difficult challenges to face, because they are symptomatic of ourselves and of culture, and so the problem becomes internalised, not externalised. However, if we are serious about tackling the myriad agents of environmental change, they are also the challenges we ought to be facing. To understand these challenges we must steer away from frameworks that look for unifying theories and answers. We must instead dive into the noise, and listen to the confusing, heterogeneous and non-human stories of environmental change, decentring ourselves from those stories. As Anna Tsing puts it, we must notice the "feral", that is, "the history making of non-humans we get involved with, particularly those outside our purposes, which are crucial to understanding the environmental challenges we face”, Tsing (2016).

This paper begins with a brief introduction to the frameworks of actor-network theory and multispecies storytelling. We discuss how together, these frameworks help shift perspectives of the environment from an exterior entity which we can control, towards something that we are always already a part of, not the centre of, but mutually entangled with and co-produced alongside. Drawing from Jennifer Gabrys' concept of non-human organisms as environmen- 
tal media, we explore alternative (and artistic) methods of articulating environmental change, focusing on the internally lived experience of non-humans outside our purposes, with an aim to render a joint environmental citizenship with non-human species. The paper then presents my own artistic intervention, Feral Robotic Birds, bird-like machines that catalogue and vocalise echoes of their changing sonic environments over time, through manipulated birdsong. Treating the lived experiences of birds as a stage onto which environmental change is playing out, and their songs as embedded in these changes, we reflect on how Feral Robotic Birds as an art/sound-work expresses environmental change, and helps us hear our own implications in those changes. We also consider the potentials of sound more broadly as a medium for engaging with non-human stories of environmental change, as well as its potential in conservation.

\section{ACTOR-NETWORK THEORY AND THE BUILDING OF LANDSCAPE}

How might we begin our dive into the noise, to think about the landscape of environmental change in terms of these heterogeneous, potentially non-human stories? Actor-network theory is a way of thinking topologically, that could serve as a useful lens. It encourages us to think not in terms of spatial dimensions or surfaces, but in "nodes that have as many dimensions as they have connections", Latour (1996: 370). The landscape becomes not an enclosed area, or system, subject to external forces, but instead boundlessly arises from multifarious networks of interacting bodies/matter. An object, or a someone existing in an actor-network is not dissimilar to how an object might exist in the context of a machine learning algorithm, where the object would inherit not a three dimensional space, but a vectoral one, with as many vectors as there are features to be quantified, and whose values decide the distance and relatedness to other objects in the corpus. Actor-network theory also ascribes liveliness, that is, the ability to interact/be affected by/change, to all entities in an environment (actors), and explores the chaotic interactions (network) that take place between them. No longer rooted in space or time, but rooted in connections across space and time, the landscape becomes an ever-evolving dynamic stage that the actors and the network both form, and are formed by ("work" being an important part of net-work, as the landscape is constantly the product of work done by entities within it).

If we follow actor-network theory to its extreme, the landscape does not actually exist, because there is nothing that is not the actors or the network between them. Paradoxically also, the forces from which landscapes emerge (the actors and the network) exist only as abstract concepts and not in themselves, for they too are always emerging from the landscape, produced by it whilst also producing it. It is not to say that we must always take these extremes literally, but that actor-network theory, as a lens, can help us see that entities and social forces do not exist concretely in and of themselves, but that bodies and worlds are at all times co-producing one another.

This conception of nature echoes Deleuze and Guattari's cultural theory of the rhizome, which "resists the organizational structure of the root-tree system", and takes instead the rhizome, characterized by "ceaselessly established connections between semiotic chains, organizations of power, and circumstances relative to the arts, sciences, and social struggles", Deleuze \& Guattari (1987: 6). Through actor-network theory we can start to see the unpeeling of the natural landscape as an exterior entity upon which we act, and instead see our entanglement, in 
it, from it, with it, as it. Importantly too the distinction between natural and cultural landscape is eroded, because everything is essentially something produced (humans being just one of many actors in this network of production). Cultural and natural become wrapped up as one, removed from distinctions of created-by-nature and created-by-human. Both become stranger, entangled, the result of a cumulative history of work by multiple actors.

\section{DECENTERING OURSELVES THROUGH MULTISPECIES STORYTELLING}

Actor-network theory's capillary-like nature echoes Donna Haraway's engagement with multispecies storytelling through "string figures" and "speculative fabulations" (SF); see Haraway (2016: 10). A difference between them lies in that actor-network theory specifically "does not attempt to fill in what is in between the filaments", whereas SF encourages us to think about our situated and meaningful connections, particularly with non-human actors:

Passing patterns back and forth, giving and receiving, patterning, holding the unasked-for pattern in one's hands, response-ability; that is core to ... staying with the trouble in serious multispecies worlds. Becomingwith, not becoming ... is how partners are rendered capable. Haraway (2016: 12)

If we extend actor-network theory to observe between those filaments that exist here and now, through Haraway's concept of becoming-with multispecies kin, we can think of actor-network theory not just in terms of an historical apparatus of the actor-network, but also as a potential-actor-network, where bodies, entanglements and stories are at every moment potentially becoming told. As actors with response-ability, we are implicated in rendering what becomes and what doesn't in this potential-actor-network (in other words, our ability to respond as actors in the network gives us a responsibility). We are all, along with our multispecies partners, joint citizens of the landscape, of ecologies and of the environment. How might we articulate environmental and ecological change so as to render this joint citizenry and kin-ship visible?

\section{SENSING AND ARTICULATING ENVIRONMENTAL MEDIA}

In 2011, during a fieldwork residency in Lapland exploring how "environmental monitoring articulates distinct practices and politics of environmental citizenship", Jennifer Gabrys considered "how creative and community-based monitoring projects might differently attend to expressions of climate change as they occur through connections made across entities and milieus", Gabrys (2013:112). Mentioned are examples that monitor complex and lived relations between more conventional variables, such as the atmometer, which emulates the experiences and processes of transpiration within a plant, Gabrys (ibid: 127), or the Exchange for Local Observations and Knowledge of the Arctic (ELOKA), which documents traditional knowledge and community monitoring activities, Gabrys (ibid: 124). Interestingly, multiple examples of non-humans as expressions of environmental processes are also included, such as the Pika, whose stress hormone metabolites in faecal samples help monitor habitat changes in mountainous regions. Like technologies, organisms like Pikas are assemblages of their environments, quite literally inheriting, working through and living with changes. Gabrys calls them "environmental media of sorts" to describe this phenomenon. If we think of organisms as environmental 
media i.e. snapshots (or reports) of the environment through time, they can become interesting variables to study, because they are simultaneously the multi-dimensional nodes actor-network theory proposes we think with, but also frame non-human kin as environmental citizens, whose internally lived experience is the stage onto which environmental change plays out.

There are many art-science-works like Elaine Gan and Anna Tsing's “A Fungal Clock”, Gan and Tsing (2012), and Amelia Black's "Amphibious Architecture", Black (2009), that use the idea of organisms as environmental media, whilst approaching multispecies thinking in a way of care and attention towards non-human modes of being, framing us as listeners (and re-actors), as opposed to the anthropocentric role of controllers or utilizers from which we so often approach our non-human counterparts. In "A Fungal Clock" we experience multiple modes of time/potentiality, intention/un-intention, between pine, oak, humans and matsutake mushrooms. In "Amphibious Architecture" both human and non-human inter-actors are rendered as environmental citizens, where humans can "text fish" and receive information on water quality, fish numbers and human interest, thus engaging people in perceiving a shared citizenry with urban non-human kin. Sound-artist/ecologist Jana Winderen interprets the idea of organisms as environmental monitors by listening to them. On her piece "The Listener" Winderen says that, "if we identify the sounds of specific species, we can understand river health through focused listening", Winderen (2016). Through technology she extends our acoustic horizons and amplifies inaccessible sounds of landscapes, such as glaciers in her piece "Isolation/ Measurement", Winderen (2010). Sound here can be understood as an abstract, non-specific and simple language shared across insects, icebergs and humans, not unlike the "infralanguage" Latour refers to in "On actor-network theory", Latour (1996: 377). Artistic modes of practice can clearly articulate environmental change as something experienced by humans and non-humans alike, on one level attending us to those differing non-human experiences, whilst on another level rendering them as shared between us, as joint environmental citizens.

\section{ARTISTIC INTERVENTION - FERAL ROBOTIC BIRDS}

In the rhizomatic spirit of actor-network theory and SF, I wanted to explore non-humans as environmental media, though not as pollution detectors or to answer other human enquiries, but simply to listen to non-human environmental experiences, outside our purposes as such. Adapting the title of Natalie Jeremijenko's pollution detecting "Feral Robotic Dog", Jereminjenko (2006), I named my intervention Feral Robotic Birds. It's a speculative artwork that expands notions of environmental modelling and imagines the future of birdsong, through listening to and registering current environmental soundscapes, and vocalizing the histories of those soundscapes through manipulated birdsongs.

Part of the inspiration for the project was a radio podcast on starlings living in a barn, who were reported to be mimicking an engine, BBC Earth (2017). The engine, long disused, must have been mimicked by the starlings' ancestors, who then passed this strange song through the generations. I began to think about the starlings as both sonic reflections of their environments, but also artistic agents, inspired by their surroundings and expressing a cultural change in the environmental landscape, through song. I say cultural change referring to the many ways birds' lives and relations to one another change with environmental change. For instance the fact that birds now sing louder to compete with road traffic and aircraft noise, that fewer birds are 
being born due to partners not hearing one another's songs, or are re-housed and dispossessed by building development projects, can all be understood as cultural changes, created by environmental changes we as actors constitute. Such interactions between birdsong and mechanical sound have been explored by artist Alexandra Ginsberg, who uses generative adversarial networks to create machinic birdsongs with a critical angle to the effects of human-made sounds on birds, Ginsberg (2019).

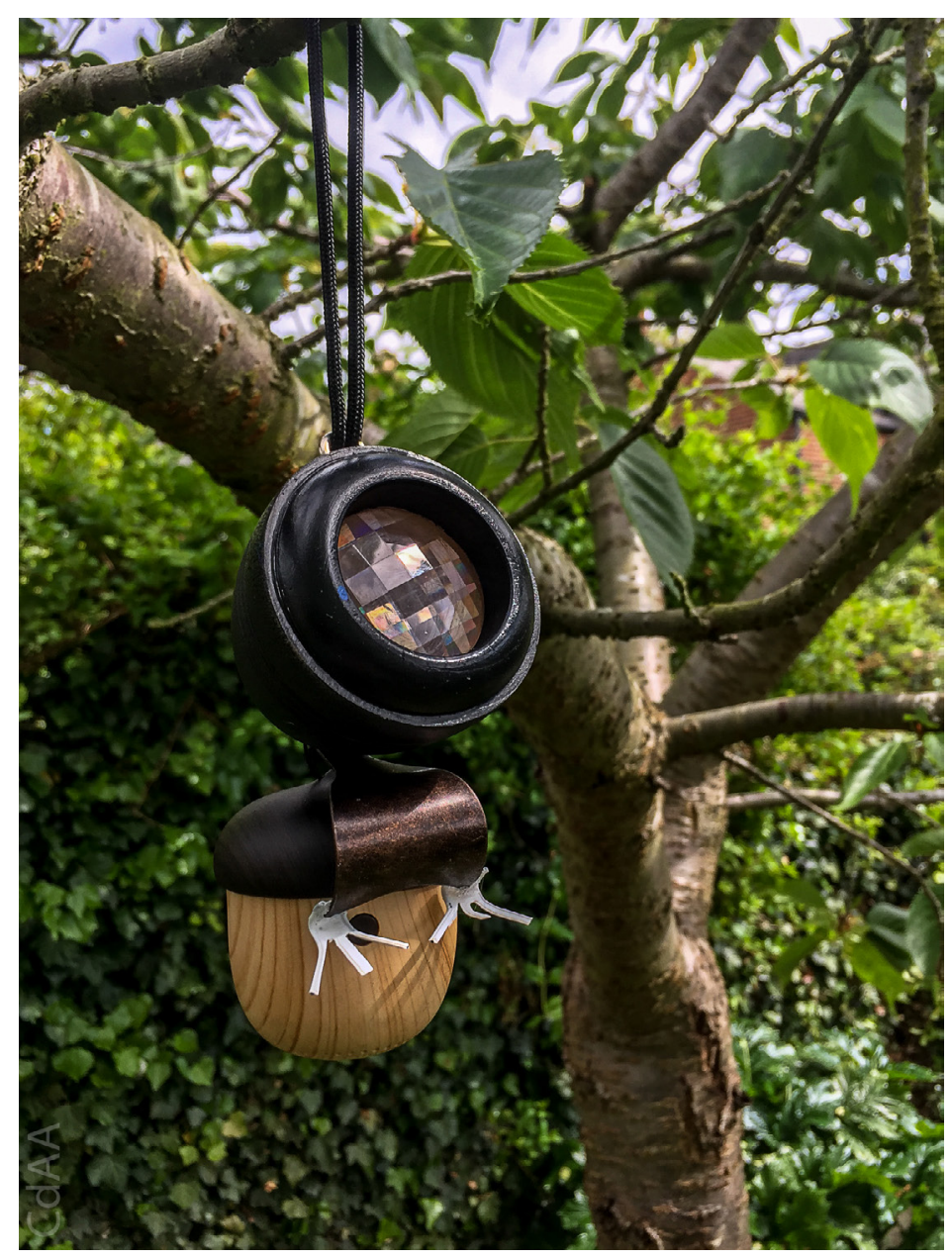

Feral Robotic Bird in tree. Photo by the author.

The starlings and the engine shows how sound can map a history of relations. Just as geological sedimentation leaves historical traces, so too will echoes of us and the worlds we engender exist in future birdsongs. Similarly, echoes of the past exist in birdsong now. Analysis of ice core's composition yields climatic information dating back 800,000 years. What could the history of sonic relationships existent in multispecies voices tell us about the past, if only we had the means to dissect and understand them? By cataloguing the histories of their sonic environments and gradually incorporating them into each new vocalization, Feral Robotic Birds encourage us to consider sound as historical record, whilst simultaneously being an artistic speculation of future birdsongs and environments. Feral Robotic Birds are also preservationists. Like Alan Lomax's field recordings of American folk songs, which led to UNESCO's "Appeal for Cultural Equity", defending every culture's right to safeguard, express, and develop its artistic heritage, Feral Robotic Birds are archives of birdsongs. When these recognizable songs begin to change, we are encouraged to consider the songs as a developing heritage of cultural artefacts to be preserved. 
This (https://www.youtube.com/watch?v=NcQUgbvJ3A8) is a video of Feral Robotic Birds in action. Part 1 shows the bird's initial calls (a nightingale call). Part 2 shows the bird after listening to its environment for about an hour. Part 3 shows a modification I made to the calls, by slowing them down. Nightingales' auditory recall is faster than ours, meaning they perceive each other's songs slower than we do. By slowing them down, we hear them closer to how a nightingale would hear them, and I think this demonstrates how much information is actually in the calls; they sound very much like a language. Part 4 shows an experiment where the Feral Robotic Bird has been listening to Thelonius Monk.

\section{TECHNICAL AND AESTHETIC CONSIDERATIONS}

My prototype, presented here, takes nightingale songs as its initial repertoire. Nightingales are birds I've worked musically with before on a research project exploring human migrant stories and nightingale song, and so I had field recordings of them I could use for this prototype. The technical aspects are handled in a Max/MSP patch. Although a future version of this project could involve machine learning to analyse archived recordings of birdsongs changing over time, and to allow the robot to adapt to its sonic environment, I took a simpler approach for the purposes of this project. The patch contains five initial nightingale calls, triggered at semi-random intervals. The character of the surrounding environment is recorded and incorporated into the birdsong through convolution (explained below), via a gradual process. The longer you leave a Feral Robotic Bird in its environment, the more it begins to adapt its call in response to the sonic history of that environment.

Convolution multiplies the frequency content of two signals over time, producing an impression where one signal sits inside another. An issue that occurred was that after a few hours, Feral Robotic Birds became very noisy, and began to simply sound like the effect of convolution itself. I filtered out background sounds below certain amplitudes and without distinct sonic profiles, and added a high-pass filter, as the convolution was exaggerating lower frequencies. I also added an automatic volume controller that compensates in response to the loudness of the bird's call and the environment it listens to, so that the convolution process does not produce a birdcall that becomes too loud or too quiet when left for long periods of time.

In the vein of "public amateurism", Costa (2008: 173), of artists doing science, my bird is a DIY stitching-together of household objects. The head is a small painted kaleidoscope, echoing a camera lens, and giving a machine-like, even surveillance-like appearance. The feet are cut from tags of rice cake packets, attached to a picture-frame hook. A Bluetooth speaker, which is connected to a laptop containing the Max/MSP patch, functions as both the microphone (or ears of the bird) and speaker (mouth of the bird). A string attached round a hook allows the bird to be hung from branches or other objects. It can also stand on surfaces, or sit in the lid of some shoe spray. 


\section{THe Power of Sound AS A Sensory Medium}

Sound is one step away from language (sounds in an airport, or the sound of elevators in hotels, are already language), but once you are in the street, most sounds are untamed, they are in the act of becoming. Sala in. Toop (2019: 170)

For monitoring multispecies actors-in-networks/networks-in-actors, with a power to broaden our conception of who/what can count as an actor, or environmental citizen, I chose sound as my sensor, and this requires some explanation. Sound is not a text, or a visual object, but a relational mapping through fluctuations in air. It passes through circumstances, traversing micro-macro scales, echoing and reflecting states of landscapes, and recomposing itself in and out of beings through voice, and through time and space. Thus sound as voice can be understood as a deconstruction of individuated expression and of self, and instead an entity unto its own, a relational history of sonic reflections, prompted and provoked across multiple bodies, entities and experiences. You might say, "I am hungry", but the bacteria in your gut make your belly rumble. Simultaneously however, sound reterritorializes individuals in space, it's a "here I am" of something there. It's this double-edged quality of sound as both relational history, but also the culmination of that history into the seeming expression of oneself as voice exits the body, that makes it interesting as environmental media. As Dominic Pettman puts it, sound is an "aural punctum" with "the potential to pop or connect...ontological bubbles", Pettman (2017: 75), and furthermore voice is a "sonic exploration of ontological conditions" and "[tests] the world and one's location, role and value in it", (ibid: 54).

Through sound's reflexive nature, I wanted to ask how non-human purposes relay back to us, as response-able environmental citizens. With the help of machine-kin, I explored a situated story of environmental change through the vocalizations of birds, whilst also speculating on future vocalizations and environments. Realizing sound's ability to shift ontologies and pop/ connect ontological bubbles as Pettman testifies, I thought an intervention here was important, in:

- Continuing the sensing of environmental change through organisms as environmental media, and its trajectory towards concretizing notions of shared environmental citizenship with non-human kin.

- Showing how, through creative listening and imagining of future environments, the felt and lived non-human experiences of environmental change can be rendered visible (and audible), along with the connectedness of our own actions (response-ability) with respect to these non-human experiences.

\section{Potentials of SOUND in ECOlogical ConTEXTS}

Soundscape ecologist Bernie Krause demonstrated sound's power to tell stories of ecological change. In his "Great Animal Orchestra", various sonic niches of frequency and time are filled with living sounds, compared to recordings taken a decade later after deforestation. Silence is a powerful indicator of effects on biodiversity. However it is fair to say that sonics have not made the cut as environmental indicators at the scientific level (not one of the 50 essential variables used to inform the UNFCCC and the IPCC is sonic, for instance). Understandably, this is because, although sound is intuitively meaningful to us when listening to it with known 
context (as Krause did), the feasibility of deriving meaning from large datasets of sound on global scales is extremely difficult.

There are those who are attempting to begin the process of involving sound in environmental monitoring however, whilst maintaining an engagement with human and non-human environmental citizenship. Over the past few years, Dan Stowell's on-going research into automatic birdsong recognition through machine-learning has resulted in an app, Warblr, that allows people to engage in identifying bird species present in an area through their songs from their smartphones. The algorithms focus on species categorization, as opposed to any expressive content within the birds' vocalizations. However sonic analysis is relatively new in machine learning, and unlike the further developed analysis of human-made sounds, where machines are able to understand, to an extent, the verbal and emotional content of what is being said, analysis of non-human-made sounds isn't there yet.

An issue that makes analysing sound with machine learning difficult, as Stowell describes, is that unlike visual objects, sonic objects are not contained as continuous spatially entities, Stowell (2020). When presented on a spectrogram, they spread across one other, due to fundamental frequencies existing above the main frequency of any occurring sound. The language and infrastructure around sonic analysis still needs attending to. This is where, when using a framework like actor-network theory, which, as opposed to a "descriptive vocabulary", rather "opens, against all a priori reductions, the possibility of describing irreductions", Latour (1996: 375), we must take care to navigate the "untamed" and abstract qualities of sound described by Sala. It is not that we should stop short of knowing these abstract qualities, but rather take the opportunity not to anthropomorphise (or anthropo-sonify) this untamed realm; to remember that it is in fact these qualities of abstraction that give sounds power in cross-species storytelling, and to allow other (non-human) languages to grow from them, be they bird languages, machine-learning languages, etc.

Stowell describes how answering the questions, "are there any birds here?" and "which birds are present?" are equally difficult to ask with machine learning, despite the former seeming easier, Stowell (2020). From this we can grasp the leap between machine logic and human thinking, but also the exciting potential of asking seemingly difficult questions like, "what are the birds saying?" For now the app is useful in engaging people in environmental monitoring, and for conservation, as audio can be archived to detect changes in populations and migration patterns, particularly where birds can't be seen but are easily heard. The project also aims to help biologists understand the evolution of bird language, and identify social cues of various groups/ individuals. This is part of a wider area of research into bird (and animal) communication (such as research into Japanese Great Tits' use of compositional syntax to produce various meanings from individual calls/notes, Suzuki et al (2016: 1-7)).

However as feral thoughts teach us, communication may already be occurring on the peripheries of technology. Hanna Wirman's communicative experiments with orangutans through gaming consoles showed that the multispecies language of play manifested itself not through the consoles, but through the orangutans grabbing Ethernet cables, cage doors and human hands. Although many times stronger than humans, the orangutans respected "the physical capabilities of the other." Thus a "meta-language" (a non-verbal language based on mutual recognition of physical capabilities) formed here between orangutans and humans, "establishing the other in relation to oneself", Wirman (2013:2-3). 


\section{REFLECTIONS ON FERAL ROBOTIC BIRDS}

There are of course limits to my own project in an ecological or environmental context. Although it helps us think about frictions between ourselves, birds, songs and environmental change, it is not able to inform normative guides to action like the 50 variables informing the IPCC do, and does not suggest we stop measuring such variables. But through reframing the story of environmental change through sound, Feral Robotic Birds gets us to think about an extended sense of self and environmental citizenry, that stretches across species boundaries and machines, and frames environmental change as occurring internally as well as externally. Furthermore, Feral Robotic Birds are sentinel species-machines that tell us something about themselves, and whose direct concern is not human wellbeing or action (for more on sentinel species, see Gramaglia (2020)). They get us to think about the collateral of our behaviour, and the technologies we create on our non-human kin. They help us see that the natural world is not something external to be measured, but something we are always already part of, reminding us of our response-ability. During the COVID-19 pandemic, many have remarked on the prevalence of birdsong in cities, where normally there would be traffic and other more traditional urban sounds. For now, SARS-CoV-2, an actor itself in the network of our current landscape, has made us consider our sense of place amongst technological noise and birds.

The songs are not a unified explanation of environmental change or of nightingale's experiences of that change. Sound doesn't tend towards linear causes, but trickles sideways, reflecting, re-appearing, and dying. As Francis Alys describes, sound is "a certain way of delaying the narration or postponing the conclusion... a progression always, but a kind of going back or forth". He says, in a way that reflects the co-production of actor-networks and landscapes, how the sonic nature of dynamic cities enforces adaption, where once the urbanist had to "plan ahead the expansion of the city" it is now "to react to given situations of spontaneous urban growth...there is an absence of any master plan", Sala in. Toop (2019: 174). This mirrors the turn of 20th century composers and musicians like Cage and Lucier, de-centring themselves from controllers of sound, to listeners and collaborators. Science that works with organisms as environmental media could take this on board. Sound talks back at you, and it's this reflexive nature of sound, leading us as responders to it, as a means to a conversation with the environment, that I wanted to capture with my artwork.

Our cultural impressions of nightingales are also important as they inform how we hear and understand Feral Robotic Birds. Historically nightingales have represented foreboding and change, and their songs metamorphose in Feral Robotic Birds just as Procne metamorphoses into a nightingale in "Tereus", forever mourning in the forest. As in Keats' "Ode to a Nightingale" both the ethereal, already there-ness, of nature can be heard in Feral Robotic Birds, but so too can its fleetingness, the pace of change and fragility of existence. Today nightingales are known to be migrants, each year they fly from Africa to Europe, and are met at the borders with guns and mist nets for hunting. In the UK, their habitats (low scrubland) are destroyed by military training grounds and housing development projects, Birkhead (2018). It is through the "untamed" abstract qualities of sound Sala referred to, the intonations, the "infralanguage", the music, that we have come to understand nightingales as part of our cultural landscape in stories and in poems, and have been able to hear our existence reflected in their songs. Nightingales sing hundreds of highly complex, socially learned and individually adapted songs. Through actor-network theory we are not required to partition those songs as being the individual's alone, but can treat them as the work of multiple actors, thus becoming 
sonic reflections that tell us stories about ourselves, our multispecies kin and the environments we share. Importantly, presenting their songs through the robot unhinges ideas of voice as being the individual's alone. Animal-machine-environment become interconnected actors; Feral Robotic Birds are a kind of performative becoming-with one another. An engagement with birds and their environments in this way provides insights that the hides and blinds of science do not, and informs a "deep and critical knowledge of the ecosystem....achieved through not only the scientific mode of thinking but also other forms of knowledge and perception, such as the humanities and the arts", Brown \& Timmerman (2015: 1).

\section{FERAL ENDNOTES}

An unintended outcome from Feral Robotic Birds occurred when I took my prototype on a nearby walk where some of the first nightingales had arrived to nest near an estuary in Essex. The Feral Robotic Bird seemed to encourage the nightingales to sing in competition. This probably occurs as the male nightingales, who arrive first, establish their territories through singing, before the females arrive a few weeks later. The songs are a way of negotiating territories, and are also eventually sung to attract a female. With the development of apps like Warblr and their use for conservation, I wonder if Feral Robotic Bird's ability to "bring out" the nightingales could be developed, and potentially help in counting numbers. On the other hand, it must be recognised that when placed among nightingales, Feral Robotic Birds do in fact interfere, if only a little, with the marking and competing for territories, which are a vital part of the species' continuation. Before implementing Feral Robotic Birds in any mode of conservation (or conversation), the implications of doing so would need proper advice and assessment.

As an art piece however, the communicative element of Feral Robotic Birds makes me think of potential actor-networks. Like the practice described by Shiho Satsuka, of disturbing the landscape, in the hope matsutake mushrooms might potentially grow, Tsing (2015: 302), Feral Robotic Birds digs a little into the dirt of nightingale-human-machine-environment interaction and communication. At a lecture titled "Ask the Birds" in 2018, bird scientist Tim Birkhead talked of a bullfinch he'd met who had mastered folk songs better than human whistlers, and greeted its owner with the songs more affectionately than a dog. So sonically different from their usual "squawk", this suggests extensive brain activity, and although that activity is hijacked here from its usual uses (in the typical messiness of multispecies collaboration), it suggests unknown and rhizomatic potentials in ways sound can manifest itself through non-humans and humans alike, and in the ways animals could think, act and express themselves, in worlds where we interacted more positively and fully with them.

\section{BIBLIOGRAPHY}

Allen, Casey D. 2011. "On Actor-Network Theory and landscape" Area 43(3): 274-280. Available at https://www-jstor-org.gold.idm.oclc.org/ stable/41240503?seq=1\#metadata_info_tab_contents/ 
BBC Earth. 2017. “The incredible generational memory of starlings" BBC Radio 3. Retrieved on May 4, 2020 https:/www.facebook.com/bbcearth/videos/1630414800313735/?hc_re $\mathrm{f}=\mathrm{ARR} 9 \mathrm{xP} 9 \mathrm{BBHgTEpsWbJdKxxwxfFGDBVNHyiLuiX0q0kbvoaVxPrUsHD68armT}$ tR4so6o

Black, Ameilia, 2009. "Amphibious Architecture” Toward the Sentient City Exbibition. Available at https://portfolio.ameliablack.com/

Birkhead, Tim. 2018. Ask the Birds, Lecture at. Whitechapel Gallery.

Brown, Peter G. and Peter Timmerman. 2015. Ecological Economics for the Anthropocene: An Emerging Paradigm. New York: Columbia University Press.

Costa, Beatriz D. and Kavita Philip. 2018. Tactical Biopolitics: Art, Activism, and Technoscience. Massachusetts: MIT Press.

Deleuze, Gilles and Felix Guattari. 1987. A Thousand Plateaus. Minneapolis: University of Minnesota Press.

Gabrys, Jennifer. 2013. Program Earth: Environmental Sensing technology and the making of a computational planet. Minneapolis: University of Minnesota Press.

Gan, Elaine and Anna Tsing. 2012. "A Fungal Clock: Experiments in Representing Time" Annual Meeting of the American Anthropology Association. Available at https://www. elainegan.com/fungalClock.html

Ginsberg, Alexandra D. 2019. "Machine Auguries" Somerset House. Available at https://www. daisyginsberg.com/work/machine-auguries

Gramaglia, Christelle. 2020. "Sentinel Organisms: "They Look Out for the Environment!" limm, Retrieved May 4, 2020 https://limn.it/articles/ sentinel-organisms-they-look-out-for-the-environment/

Haraway, Donna. 2016. "Playing String Figures with Companion Species.” Pp. 9-29 in Staying with the Trouble: Making Kin in the Cthulucene, Durham: Duke University Press.

Jeremijenko, Natalie. 2006. "Feral Robotic Dogs - Natalie Jeremijenko", Rhizome. Available at https://rhizome.org/editorial/2006/aug/26/feral-robotic-dogs-natalie-jeremijenko/

Keats, John. 1994. John Keats: Selected Poetry ed. Elizabeth Cook. Oxford: Oxford University Press.

Krause, Bernie and Jonathan Skinner. 2012. "THE GREAT ANIMAL ORCHESTRA: A Performance and Dialogue in Soundscape and Poetry", Harvard University. Retrieved December 18, 2020 https:/www.youtube.com/watch?v=tsEgbo1o70g/

Latour, Bruno. 1996. "On actor-network theory: A few clarifications" Soziale Welt 47(4): 369-381. Available at https://www-jstor-org.gold.idm.oclc.org/ stable/40878163?seq=1\#metadata_info_tab_contents/

Pettman, Dominic. 2017. Sonic Intimacy: Voice, Species Technics (or, How to Listen to the World). California: Stanford University Press.

Stowell, Dan. 2018. "Who's singing? Automatic bird sound recognition with machine learning" PyData. Retrieved April 28, 2020 https:/www.youtube.com/watch?v=pzmdOETnhI0/

Suzuki, Toshitaka N., Wheatcroft D. and Michael Griesser. 2016. "Experimental evidence for compositional syntax in birdcalls". Nature Communications: 1-7.

Toop, David. 2019. Inflamed Invisible: Collected Writings on Art and Sound 1976-2018. London: Goldsmiths Press.

Tsing, Anna. 2015. The Mushroom at the End of the World. New Jersey: Princeton University Press.

.2016. "Feral Technologies: Making and Unmaking Multispecies Dumps Interview with Elaine Gan, Bettina Stoet” Anthropocene HKW. Retrieved April 28, 2020 https://www. anthropocene-curriculum.org/project/campus-2016/feral-technologies/ 
Winderen, Jana. 2010. “Isolation/Measurement”, Energy Field. Retrieved December 18, 2020 https://janawinderen.bandcamp.com/album/energy-field

2016. 'The Listener' Ash International. Retrieved December 18, 2020 https://www. janawinderen.com/releases/the-listener

Wirman, Hanna. 2013. "Orangutan Play on and Beyond a Touchscreen' ISEA 2013 Conference, The University of Sydney. Retrieved December 18, 2020 https://ses.library.usyd.edu.au/ bitstream/handle/2123/9678/orangutanplaybeyond.pdf?sequence=1

World Meteorological Organization. 2020. "Essential Climate Variables” WMO. Retrieved December 2018, 2020 https://public.wmo.int/en/programmes/global-climate-observing-system/ essential-climate-variables 